\title{
Orthostatic haemodynamic responses in acute stroke
}

\author{
B Panayiotou, J Reid, M Fotherby, P Crome
}

\begin{abstract}
Summary
Little is known about orthostatic blood pressure regulation in acute stroke. We determined postural haemodynamic responses in 40 patients with acute stroke (mild or moderate severity) and 40 nonstroke control in-patients, at two days ('Day 1') and one week ('Week 1') postadmission. Following a 10-minute supine rest and baseline readings, subjects sat up and blood pressure and heart rate were taken for 5 minutes. The procedure was repeated with subjects moving from supine to the standing posture. Haemodynamic changes from supine data were analysed. On standing up, the control group had a transient significant fall in mean arterial blood pressure on Day 1 but not Week 1. No significant changes were seen on either day when sitting up. In contrast to controls, the stroke group showed increases in mean arterial blood pressure on moving from supine to the sitting and standing positions on both days. Persistent postural hypotension defined as $\geqslant 20$ $\mathrm{mmHg}$ systolic fall occurred in $<10 \%$ of either of the study groups on both days. Sitting and standing heart rates in both groups were significantly faster than supine heart rate on both days. The orthostatic blood pressure elevation is consistent with sympathetic nervous system overactivity which has been reported in acute stroke. Upright positioning as part of early rehabilitation and mobilisation following mild-to-moderate stroke would, therefore, not predispose to detrimental postural reductions in blood pressure.
\end{abstract}

Keywords: stroke; orthostatic hypotension; hypotension

Lesions of the central nervous system frequently cause disturbances of the cardiovascular and autonomic nervous systems. In acute stroke, raised levels of circulating catecholamines, ${ }^{1}$ parasympathetic system hypofunction, ${ }^{2}$ transient hypertension, and attenuated or reversed circadian variation in blood pressure and heart rate are common. ${ }^{3}$ However, little is known about orthostatic haemodynamic responses in the first few days post-stroke.

Stroke occurs predominantly in the elderly, who are prone to orthostatic hypotension because of the effects of ageing and comorbidity on blood pressure regulation. Postural hypo- tension in this age group reduces cerebral blood flow and carries considerable morbidity from dizziness, falls and injury. ${ }^{4}$ In acute stroke, cerebral perfusion is dependent on systemic blood pressure because cerebrovascular autoregulation is impaired. ${ }^{5}$ Thus, postural hypotension in acute stroke patients may further impair cerebral blood flow, increase stroke size, or hinder recovery. Data on postural hypotension in acute stroke may have implications for early rehabilitation and the use of vasoactive drugs. The objective of this study was to determine postural haemodynamic responses in the acute and recovery phases of stroke.

\section{Materials and methods}

\section{SUBJECTS}

Patients aged $\geqslant 65$ years with a mild or moderate (Canadian Neurological scale ${ }^{6}$ score $\geqslant 70$ ) acute ischaemic stroke (WHO definition) were considered for the study. Those with haemorrhagic stroke confirmed on computed tomography (CT) scanning or suspected clinically by the presence of headache, neck stiffness and vomiting, and patients with a Guy's score ${ }^{7}$ predicting a $<90 \%$ chance of an ischaemic stroke were not included. An age- and sex-matched control group was selected from admissions without history of cerebrovascular disease. Both groups had to be conscious and cooperative, previously mobile and living at home (Modified Rankin scale ${ }^{8}$ score $\leqslant 3$ ), and have no history of dizziness or falls. As about $50 \%$ of patients with acute stroke have a known history of hypertension and are receiving antihypertensive therapy, ${ }^{9}{ }^{10}$ patients were included whether they were on antihypertensive drugs or not.

Subjects were excluded if they had coexisting illnesses which can affect blood pressure regulation (eg, diabetes, Parkinson's disease), severe ischaemic heart disease or heart failure, myocardial infarction in the previous 6 months, atrial fibrillation, evidence of dehydration, anaemia, bleeding, pneumonia, septicaemia, renal failure, respiratory failure, or pulmonary embolism. The study was approved by the local ethics committee, and informed consent was obtained from all participating subjects.

\section{MEASUREMENTS}

Both groups were studied within 3 days of admission ('Day 1'), and again 4-7 days after the first study ('Week 1'). In stroke patients, Day 1 had to be within 3 days of stroke onset. Blood pressure and heart rate readings were taken using an automatic SpaceLabs 90207 
oscillometric device (SpaceLabs Inc, Redmond, WA, USA) which meets the validation criteria of the British Hypertension Society. ${ }^{11}$ In stroke patients, the cuff was applied to the non-paretic arm. In all subjects the same arm was used for the two studies. Patients were studied before or $>2$ hours after meals on both days.

Following 10 minutes of supine rest, the mean of two successive blood pressure and heart rate readings were recorded. Single measurements of blood pressure and heart rate were then taken at 1,3 , and 5 minutes after moving to the sitting position. Following a further 10-minute supine rest and baseline measurements, the procedure was repeated with subjects in the standing position. Stroke patients who were unable to stand independently or with minimal help (eg, because of their neurological deficits), were not investigated in the standing posture. Hypotensive symptoms during the sitting or standing phases (dizziness, light-headedness, disturbance of conscious level) were noted.

ANALYSIS

Haemodynamic data

Mean arterial blood pressure (MABP) was calculated using the formula:

$$
\mathrm{MABP}=\mathrm{DBP}+[\mathrm{SBP}-\mathrm{DBP}] / 3,
$$

where DBP and SBP stand for diastolic and systolic blood pressure, respectively. All haemodynamic data were entered into a computerised database. Within- and betweengroup comparisons for baseline parametric data were made with the Student's paired and unpaired $t$-tests, respectively, the significance level being $5 \%$. Paired $t$-tests were used in each group to compare 1-minute sitting and standing with supine haemodynamic parameters. Haemodynamic changes from supine to 1 minute (after transformation to percentage changes to allow for baseline inequalities between the stroke and control groups) were compared between the two groups with unpaired $t$-tests. Two-way analysis of variance (ANOVA) was used in each group to assess whether haemodynamic changes from baseline differed significantly at 1,3 , and 5 minutes of sitting and standing. Pearson's correlation coefficient was used in both study groups to assess the correlation between postural haemodynamic changes and (a) pre-stroke Modified Rankin score, and (b) Canadian Neurological scale score in stroke patients. Non-parametric data were compared between the stroke and control groups with the Mann-Whitney U test.

\section{Antihypertensive therapy}

Postural haemodynamic changes were also analysed within the stroke and control groups for patients on or off antihypertensive drugs. Paired $t$-tests were used for changes from baseline to 1 minute in treated and untreated patients. The haemodynamic changes at 1 minute were compared between treated and untreated patients within each group using unpaired $t$-tests. Comparisons of non- parametric data between treated and untreated patients were made with the Mann-Whitney U test.

\section{Postural hypotension}

The incidence of postural hypotension as defined by $\geqslant 20 \mathrm{mmHg}$ systolic drop was determined, and the chi-square for trend test was used to assess changes with time in each group. Between-group comparison of the frequency of postural hypotension was made by the chi-square test.

\section{Study power calculation}

It was calculated that, given that the SD of the supine to standing SBP difference in elderly hospitalised patients is $\pm 10 \mathrm{mmHg},{ }^{12} 28$ subjects would be needed to detect a withingroup orthostatic SBP change of $\geqslant 6 \mathrm{mmHg}$ with a power of $80 \%$ at the $5 \%$ significance level. To show a difference of $\geqslant 6 \mathrm{mmHg}$ between stroke and control subjects with the same power and significance level, 40 subjects would be needed in each group.

\section{Results}

SUBJECTS

Forty stroke patients (21 male), mean age 76 years (SD 7.3, range 65-92), were studied, of whom 35 had hemiplegia or monoplegia, and five had only facial paralysis, language and/or swallowing impairment. Their median Canadian Neurological scale score was 90 (range 70-105) and Modified Rankin score was 2 (range 0-3). A CT scan was performed in 18 patients $(45 \%)$ in whom cerebral infarction was diagnosed.

The 40 control patients (20 male) had a mean age of 75 years (SD 6, range 65-90) (versus stroke group, $\mathrm{p}>0.05$ ), and premorbid median Modified Rankin score of 2 (range $0-3)$. Their presenting diagnoses were: exacerbation of chronic bronchitis (14), angina (five), arthritis (four), musculoskeletal chest pain (three), two each with thrombophlebitis, calf deep vein thrombosis, mild left ventricular failure, irritable bowel syndrome, constipation, and depression, and one each with polymyalgia rheumatica and tension headache.

\section{ANTIHYPERTENSIVE THERAPY}

Twenty-three of the stroke and 15 of the control patients were admitted on antihypertensive agents, including drugs given for other indications but with known antihypertensive properties (eg, angiotensin-converting enzyme inhibitors and calcium antagonists). Antihypertensive agents were withdrawn in some patients on admission, and during the period of study $19(48 \%)$ stroke and $13(33 \%)$ control patients received antihypertensive drugs $(p>0.05)$. The median number of antihypertensive drugs taken per patient was 0 (range $0-2)$ in the stroke group and $0(0-2)$ in the control group ( $\mathrm{p}>0.05)$.

There was no significant difference within the stroke or control group between treated and untreated patients as regards age (stroke group: $75 \pm 7$ vs $78 \pm 7$ years; control group: $76 \pm 5$ 
Table 1 Postural blood pressure $(\mathrm{mmHg})$ and heart rate changes $( \pm S D)$ in stroke patients and controls

\begin{tabular}{|c|c|c|c|c|c|c|c|c|c|c|}
\hline & \multicolumn{5}{|c|}{ Stroke patients } & \multicolumn{5}{|c|}{ Control patients } \\
\hline & $S B P$ & $D B P$ & $M A B P$ & $H R$ & $n$ & $S B P$ & $D B P$ & $M A B P$ & $H R$ & $n$ \\
\hline \multicolumn{11}{|l|}{ Day 1} \\
\hline Supine & $157 \pm 26$ & $83 \pm 14$ & $108 \pm 16$ & $75 \pm 14$ & 40 & $142 \pm 26$ & $76 \pm 12$ & $98 \pm 16$ & $78 \pm 14$ & 40 \\
\hline \multicolumn{11}{|c|}{ Change on sitting } \\
\hline $1 \mathrm{~min}$ & $-1 \pm 11$ & $5 \pm 7 \star \star \star \star$ & $2 \pm 10$ & $4 \pm 7 \dagger^{\star \star \star}$ & 40 & $0 \pm 6$ & $1 \pm 7$ & $1 \pm 6$ & $5 \pm 5^{\star \star \star}$ & 40 \\
\hline $3 \mathrm{~min}$ & $3 \pm 10$ & $5 \pm 10$ & $4 \pm 8$ & $2 \pm 7+$ & 40 & $0 \pm 8$ & $2 \pm 7$ & $1 \pm 7$ & $3 \pm 8$ & 40 \\
\hline $5 \mathrm{~min}$ & $1 \pm 13$ & $4 \pm 10$ & $2 \pm 9$ & $2 \pm 5+$ & 40 & $-1 \pm 10$ & $2 \pm 8$ & $2 \pm 9$ & $3 \pm 6$ & 40 \\
\hline Supine & $156 \pm 26$ & $83 \pm 11$ & $108 \pm 13$ & $75 \pm 16$ & 40 & $140 \pm 26$ & $76 \pm 11$ & $97 \pm 14$ & $77 \pm 14$ & 40 \\
\hline \multicolumn{11}{|c|}{ Change on standing } \\
\hline $1 \mathrm{~min}$ & $-4 \pm 16$ & $3 \pm 8$ & $0 \pm 10 \dagger$ & $9 \pm 6^{\star \star}$ & 26 & $-8 \pm 14+\dagger^{\star \star \star}$ & $-2 \pm 7+\dagger$ & $-4 \pm 8+\dagger \dagger^{\star \star}$ & $11 \pm 10^{\star \star \star}$ & 40 \\
\hline $3 \min$ & $0 \pm 16$ & $5 \pm 9$ & $3 \pm 10 \dagger$ & $6 \pm 8$ & 26 & $-4 \pm 12 \dagger \dagger$ & $0 \pm 7+\dagger$ & $0 \pm 8+t \dagger$ & $11 \pm 9$ & 40 \\
\hline $5 \mathrm{~min}$ & $1 \pm 15$ & $5 \pm 8$ & $3 \pm 9 \dagger$ & $6 \pm 11$ & 23 & $-3 \pm 16+\dagger$ & $2 \pm 8+\dagger$ & $1 \pm 9+\dagger+$ & $12 \pm 10$ & 39 \\
\hline \multicolumn{11}{|l|}{ Week 1} \\
\hline Supine & $149 \pm 21$ & $79 \pm 13$ & $102 \pm 13$ & $73 \pm 15$ & 40 & $140 \pm 25$ & $73 \pm 9$ & $95 \pm 13$ & $79 \pm 13$ & 40 \\
\hline \multicolumn{11}{|c|}{ Change on sitting } \\
\hline $1 \mathrm{~min}$ & $-1 \pm 10$ & $4 \pm 7^{\star \star}$ & $3 \pm 6^{\star \star}$ & $3 \pm 6+\dagger^{\star \star \star}$ & 40 & $0 \pm 7$ & $2 \pm 7$ & $2 \pm 6$ & $5 \pm 5+\dagger^{\star \star \star \star}$ & 40 \\
\hline $3 \mathrm{~min}$ & $0 \pm 9$ & $3 \pm 9$ & $2 \pm 7$ & $2 \pm 6+t$ & 40 & $1 \pm 6$ & $3 \pm 7$ & $3 \pm 6$ & $3 \pm 5+t \dagger$ & 40 \\
\hline $5 \mathrm{~min}$ & $-1 \pm 11$ & $2 \pm 8$ & $1 \pm 7$ & $1 \pm 6+\dagger$ & 40 & $-2 \pm 13$ & $3 \pm 7$ & $1 \pm 7$ & $2 \pm 5+\dagger+$ & 40 \\
\hline Supine & $148 \pm 21$ & $79 \pm 14$ & $102 \pm 14$ & $72 \pm 14$ & 40 & $137 \pm 26$ & $73 \pm 11$ & $94 \pm 15$ & $77 \pm 13$ & 40 \\
\hline \multicolumn{11}{|c|}{ Change on standing } \\
\hline $1 \mathrm{~min}$ & $-5 \pm 15+t^{\star}$ & $3 \pm 10 \dagger$ & $0 \pm 11+t$ & $8 \pm 14^{\star \star}$ & 36 & $-7 \pm 13+t^{\star \star}$ & $2 \pm 8$ & $-1 \pm 8$ & $13 \pm 9+\star \star \star$ & 39 \\
\hline $3 \mathrm{~min}$ & $2 \pm 13+t \dagger$ & $5 \pm 8 \dagger$ & $4 \pm 9+\dagger$ & $5 \pm 10$ & 35 & $-2 \pm 15+\dagger$ & $2 \pm 9$ & $1 \pm 10$ & $10 \pm 9 \dagger$ & 38 \\
\hline $5 \mathrm{~min}$ & $2 \pm 12+\dagger+$ & $7 \pm 9 \dagger$ & $5 \pm 8+t$ & $7 \pm 12$ & 33 & $-2 \pm 14+\dagger$ & $3 \pm 8$ & $2 \pm 8$ & $10 \pm 8 \dagger$ & 38 \\
\hline
\end{tabular}

SBP: systolic blood pressure, DBP: diastolic blood pressure, MABP: mean arterial blood pressure; ${ }^{\star} \mathrm{p}<0.05,{ }^{\star \star} \mathrm{p}<0.01,{ }^{\star \star \star} \mathrm{p}<0.001$ (paired $t$-test, 1 minute $v s$ supine); $\nmid \mathrm{p}<0.05,+\dagger \mathrm{p}<0.01$, $\dagger+\dagger \mathrm{p}<0.001$ (2-way ANOVA: 1,3,5 minutes).

vs $74 \pm 6$ years), Modified Rankin score (stroke group: 2 (range $0-3)$ vs 1 (0-3); control group: $2(1-3)$ vs 2(0-3)), or Canadian Neurological score in stroke patients (Day 1: $85(70-110)$ vs 90 (70-115); Week 1: $90(50-115)$ vs 95 (60-115)).

Baseline blood pressure and heart rate (HR) were also similar in treated and untreated patients $(p>0.05)$ in both groups, during both phases of the study: (Stroke group: Day 1: MABP $111 \pm 15$ vs $105 \pm 17 \mathrm{mmHg}$, HR $70 \pm 13$ vs $78 \pm 15$ beats/min; Week 1: MABP $104 \pm 13$ vs $101 \pm 13 \mathrm{mmHg}$, HR $70 \pm 13$ vs $76 \pm 15$ beats/ min. Control group: Day 1: MABP $96 \pm 16$ vs $99 \pm 16 \mathrm{mmHg}$, HR $80 \pm 15$ vs $78 \pm 13$ beats/min; Week 1: MABP $97 \pm 15$ vs $95 \pm 13 \mathrm{mmHg}, \mathrm{HR}$ $79 \pm 12$ vs $78 \pm 13$ beats/min).

HAEMODYNAMIC STUDIES

The stroke group was studied a median of 2 (range 1-2) days and 6 (range 5-9) days postadmission ( $\leqslant 3$ and $6-10$ days, respectively, post-stroke), and the control group 2 (range $1-3$ ) and 7 (range 5-10) days post-admission. In every patient in both groups the two studies were performed at least 4 days apart. Baseline measurements and postural responses in $\mathrm{HR}$, SBP, DBP, and MABP are summarised in table 1. None of the patients were discharged or died during the study.

\section{Supine data}

Supine blood pressure was significantly higher in the stroke group on both days $(p<0.05)$. Between Day 1 and Week 1 there was a significant fall in all supine blood pressure parameters in strokes, and in diastolic pressure in controls $(p<0.01)$. The supine haemodynamic data preceding the sitting and standing phases on each day did not differ significantly in either group.

\section{Postural responses}

In stroke patients on Day 1 (table 1), statistically significant, sustained elevations occurred in DBP on sitting up and in MABP on standing up. DBP and MABP elevations were also seen on Week 1 after sitting and standing up. The only significant change in SBP was a short-lived fall just after standing on Week 1. Heart rate increased significantly on both days at 1 minute after sitting and standing up. The rate then fell between 1 and 5 minutes post-sitting up, but remained unchanged throughout the standing-up phases.

In contrast to stroke patients, the control group on Day 1 exhibited transient falls in SBP, DBP, and MABP. At Week 1, standing DBP and MABP were unchanged despite a transient SBP fall. Blood pressures were unchanged on sitting up on both days. The patterns of heart rate responses were similar to the stroke group.

Direct comparisons of the two groups for all 1-minute data revealed a significantly greater DBP rise in stroke patients on Day 1 after sitting up $(6 \% \pm 10$ vs $1.5 \% \pm 10, \mathrm{p}<0.05)$ and standing up $(3 \% \pm 10$ vs $-2 \% \pm 9, \mathrm{p}<0.05)$.

\section{Relationship to other parameters}

There was no significant correlation between the postural haemodynamic changes and the pre-stroke Modified Rankin scores in stroke or control groups, or the Canadian Neurological scale scores in stroke patients.

Relationship to antihypertensive therapy

Statistical comparison of postural blood pressure responses at 1 minute between patients on, and those not on antihypertensive drugs revealed no significant differences in the stroke or control group. The MABP data (treated $v s$ not treated patients) are summarised below: Stroke group: Day 1 , sitting up: $-5 \pm 7$ vs $1 \pm 11$ $\mathrm{mmHg}$; standing: $-4 \pm 11$ vs $-2 \pm 6 \mathrm{mmHg}$; Week 1, sitting up: $-1 \pm 11$ vs $0 \pm 8 \mathrm{mmHg}$; standing: $-1 \pm 10$ vs $0 \pm 11 \mathrm{mmHg}$. Control group: Day 1 , sitting up: $1 \pm 5$ vs $-1 \pm 7 \mathrm{mmHg}$; standing: $6 \pm 9$ vs $3 \pm 8 \mathrm{mmHg}$; Week 1 , sitting up: $-2 \pm 5$ vs $-2 \pm 6 \mathrm{mmHg}$; standing: $0 \pm 7$ vs $1 \pm 8 \mathrm{mmHg}$. 
Table 2 Number of patients with postural systolic blood pressure fall of $\geqslant 20 \mathrm{mmHg}$ (numbers in parentheses are percentages)

\begin{tabular}{clllll}
\hline & \multicolumn{2}{l}{ Stroke group } & & \multicolumn{2}{l}{ Control group } \\
\cline { 2 - 3 } & Day 1 & Day 2 & & Day 1 & Day 2 \\
\hline Sitting & & & & \\
1 min & $2(5)$ & $2(5)$ & & 0 \\
3 min & 0 & 0 & & $1(3)$ & 0 \\
5 min & $3(8)$ & $2(5)$ & & $2(5)$ & $3(8)$ \\
Standing & & & & $7(18)$ & $6(15)$ \\
1 min & $5(19)$ & $7(19) \dagger$ & & $4(10)$ & $4(11)$ \\
3 min & $3(12)$ & $2(6) \dagger$ & & $3(8)$ & $3(8)$ \\
5 min & $2(9)$ & $1(3) \dagger$ & & &
\end{tabular}

tp $=0.05, \chi^{2}$ for trend.

\section{Orthostatic hypotension}

The frequency of patients with orthostatic hypotension is shown in table 2. Between 3\% and $19 \%$ of strokes and $8-18 \%$ of controls exhibited a systolic fall of $\geqslant 20 \mathrm{mmHg}$ (sustained or transient) during the standing phase in the two sessions. There was no significant difference within each group between the two days, or between groups on each day. Prevalence of postural hypotension fell significantly in the stroke group during the standing phase on Day 7.

\section{Symptoms}

One stroke patient developed dizziness on standing and had a systolic fall of $>20 \mathrm{mmHg}$. In all remaining patients who were unable to complete the standing phase (see table 1) no hypotensive symptoms were reported, and the reasons for the inability to stand for the full 5 minutes were the motor deficits and poor balance due to their stroke.

Five control patients reported orthostatic dizziness; in two of them it was mild and they were able to complete the study, but in three the dizziness was severe and prevented them from completing the standing phase (see table 1 ). In these three patients, one had a systolic fall of $>20 \mathrm{mmHg}$ and the others $<10 \mathrm{mmHg}$. No symptoms were recorded in the stroke or control group in the sitting position.

\section{Discussion}

In agreement with previous studies of nonstroke elderly admissions, ${ }^{13}$ the control group exhibited an orthostatic blood pressure fall soon after admission. A week later, stability of mean blood pressure on standing was restored in these patients. Unlike the control group, acute stroke patients had significant elevations, rather than falls, in diastolic and mean blood pressures when they moved from the supine to the sitting or standing postures. The incidence of sustained postural hypotension defined as $\geqslant 20$ $\mathrm{mmHg}$ systolic fall was $<10 \%$ in both groups.

Sudden orthostasis causes an immediate blood pressure fall because of peripheral venous pooling and reduced cardiac output. Normally, feedback from baroreceptors quickly leads to sympathetic system activation and parasympathetic inhibition, with compensatory rises in heart rate and peripheral resist- ance. This restores cardiac output with a fall or no change in systolic pressure, a rise in diastolic pressure, and consequently a mean arterial pressure similar to the supine level. ${ }^{14} \mathrm{~A}$ haemodynamic steady-state is normally reached within one minute. ${ }^{15}$ The same pattern is seen in healthy adults and the elderly, although in the latter the tachycardia and diastolic rise can be less pronounced. ${ }^{14}$

The rise we observed in postural blood pressure post-stroke is likely to be due to sympathetic nervous system activation. Indices of baseline sympathetic nervous system activity, eg, serum and urinary catecholamines, are raised in the first week post-stroke. ${ }^{16}$ This has been attributed to stress from acute illness, effects of cerebral tissue damage, and ischaemia of autonomic nervous system centres. A positive correlation between the degree of sympathetic overactivity and the level of resting blood pressure in acute stroke has also been reported. ${ }^{16} \mathrm{~A}$ significant association has also been found between the extent of sympathetic activity and attenuation of the physiological blood pressure fall at night. ${ }^{17}$

However, only a few studies have sought to determine postural haemodynamic responses following stroke. Korpelainen et $a l^{2}$ performed passive orthostasis $\left(90^{\circ}\right.$ tilting, up to 7 minutes) in 40 ischaemic stroke patients (mean age 51 years) within 10 days of onset, but did not find a significant change in blood pressure. While physiological responses to active orthostasis (standing up) and passive orthostasis $\left(\geqslant 70^{\circ}\right.$ tilting) are usually similar, ${ }^{18}$ Korpelainen et al studied much younger patients, in whom resting sympathetic activity ${ }^{19}$ and its response to orthostasis ${ }^{20}$ are known to be significantly less than in the elderly. Robinson et $a l^{11}$ studied nine elderly patients and eight matched non-stroke control subjects $\left(60^{\circ}\right.$ head-up tilt) and found a statistically significant blood pressure fall in stroke patients compared with control subjects. However, that study was performed between one and three weeks post-stroke rather than within the first week. Farnworth et al $l^{2}$ measured blood pressure in the sitting position and after 1 minute of standing in 15 elderly stroke patients (postacute phase) who were receiving rehabilitation at a Day Hospital, and found a non-significant blood pressure rise of $12 / 10 \mathrm{mmHg}$. Naver et $a l^{23}$ performed head-up tilt $\left(80^{\circ}\right.$, for 5 minutes) in 23 patients (mean age 50 years) between 8 and 48 days post-stroke (mean 18 days) and a matched group of healthy subjects. They reported that after 30 seconds the stroke patients had a significantly greater fall in SBP than controls (9 vs $1 \mathrm{mmHg}, \mathrm{p}<0.05$ ), without a significant change in DBP. No further change was seen in the control group, whereas in stroke patients blood pressure continued to fall and stabilised at 2.5 minutes (magnitude of the blood pressure fall was not given). Johnson et $a l^{13}$ tested 100 unselected elderly acute admissions and found $>20 \mathrm{mmHg}$ systolic fall within 2 minutes of standing in 17 patients. All of the latter patients were reported to have 'evidence of cerebrovascular disease' but it was not stated how many had an acute stroke. Palmer ${ }^{24}$ also 
reported significant orthostatic blood pressure reduction in chronic stroke. The present study was within the first 3 days of stroke onset, and showed orthostatic blood pressure stability or rise. This is in contrast to the above studies which were predominantly in sub-acute and chronic stroke, ${ }^{213}{ }^{21-24}$ and showed either orthostatic blood pressure reduction or no significant change. Detailed investigation of chronic stroke patients has previously revealed sympathetic nervous system hypofunction, ${ }^{25}$ whereas in the first few days post-stroke, sympathetic nervous system hyperactivity has been found. ${ }^{16}$

In both study groups, we found no difference in orthostatic blood pressure responses between those on antihypertensive drugs and the remaining patients. Similar results were reported recently in elderly non-stroke inpatients. ${ }^{26}$ However, prospective studies of patients already on antihypertensives have shown that drug withdrawal leads to a significantly reduced prevalence of orthostatic hypotension as defined by $\geqslant 20 \mathrm{mmHg}$ systolic fall. ${ }^{27}$

In the present study, we sought to investigate ischaemic strokes, which account for $85 \%$ of all strokes. While haemorrhagic strokes were excluded by CT scanning in $45 \%$ of patients, the remainder were included only if the Guy score predicted $>90 \%$ chance of nonhaemorrhagic stroke. Such scoring systems have limitations, and $10 \%$ of those diagnosed as having had ischaemic stroke could have had haemorrhagic stroke. ${ }^{28}$ Thus, about two patients in the present study might have been misclassified. Even though acute haemorrhagic strokes have a greater impact on the autonomic nervous system and blood pressure level than ischaemic types, ${ }^{29}$ this small number would not have significantly influenced our findings. Also, we did not differentiate ischaemic strokes into their different sub-types, ie, cortical, subcortical, lacunar, or brainstem. Recent evidence suggests differences between ischaemic subtypes in their effect on sympathetic nervous system function and 24-hour blood pressure profiles. ${ }^{17}{ }^{30}$ Another methodological aspect of our study was the use of intermittent rather than continuous haemodynamic recording. Although the latter method enables beat-tobeat haemodynamic monitoring, it is more prolonged changes which are of clinical importance. ${ }^{31}$ Intermittent sphygmomanometry has been found to be adequate for this purpose, ${ }^{31}$ and recent comparative studies of the two methods showed high accuracy of sphygmomanometry in detecting postural hypotension. ${ }^{32}$

1 Myers MG, Norris JW, Hachinski VC, Sole MJ. Plasma norepinephrine in stroke. Stroke 1981;12:200-4.

2 Korpelainen JT, Sotaniemi KA, Suominen K, Tolonen U, Myylyla VV. Cardiovascular autonomic reflexes in brain infarction. Stroke 1994;25:787-92.

3 Panayiotou BN, Taub NA, Fotherby MD. Twenty-four-hour blood pressure profiles following stroke. Blood Press Monitor 1996;1:409-14.

4 Kenny RA. The trauma of a fall. In: Kenny RA, ed, Syncope in the older patient: causes, investigations, and consequences of syncope and falls. Oxford: Chapman and Hall Medical Publishers, 1996; pp 11-14.

5 Meyer JS, Shaw TG. Cerebral blood flow in ageing. In: Albert ML, ed, Clinical neurology of ageing. New York: Oxford University Press, 1984; pp 178-96.
The findings of the present study suggest that upright positioning as part of early rehabilitation in most patients with mild or moderate acute ischaemic stroke is unlikely to cause detrimental falls in orthostatic blood pressure and cerebral blood flow. However, a minority of patients did exhibit a significant postural fall, even on sitting up. Also, we excluded patients whose stroke was severe, who had other coexisting severe illness, or were previously very disabled. Such patients have a greater risk of postural hypotension. ${ }^{33}$ Furthermore, significant reductions in cerebral perfusion can occur even with small reductions of systemic pressure, especially in susceptible individuals, eg, those with carotid artery or intracranial arterial stenoses. Randomised acute stroke trials of neuroprotective agents with co-existing hypotensive properties, reported that resting systolic reduction as small as $\leqslant 10 \mathrm{mmHg}$ was associated with significantly worse neurological recovery and survival, especially in older patients. ${ }^{34}$ As postural hypotension may exacerbate cerebral damage, it is important to identify the subgroup of acute stroke patients who are at risk by routinely taking supine, sitting, and/or standing blood pressures. In a recent investigation of blood pressure evaluation in acute stroke, it was disappointing to find that postural readings were rarely carried out in the first week post-stroke. ${ }^{35}$

Once patients at risk are identified, measures aimed at minimising postural hypotension can be taken. They include maintenance of adequate hydration, withholding certain drugs (eg, vasodilators, anticholinergics, sedatives), and avoiding fast changes in posture. Physical exercise programmes which can be used before full mobilisation, have been shown to ameliorate postural hypotension, ${ }^{36}$ although this has not been specifically assessed in acute stroke patients. In the present study, patients were not investigated soon after meals in order to avoid the confounding effect of the normal postprandial blood pressure fall that occurs in the elderly. ${ }^{37}$ Postprandial hypotension is detrimental to cerebrovascular blood flow, ${ }^{37}$ especially in people with existing cerebrovascular disease. ${ }^{38}$ It is therefore prudent to also minimise physiotherapy and postural changes in the first couple of hours post-prandially. ${ }^{39}$

We thank Mr R Sheaf of Boehringer Ingelheim Ltd for the loan of the SpaceLabs 90207 device.

6 Cote R, Battista RN, Wolfson C, Boucher J, Adam J, Hachinski V. The Canadian Neurological scale: validation and reliability assessment. Neurology 1989;39:638-43.

7 Celani MG, Ceravolo MG, Duca E, Minciotti P, Caputo N. Was it infarction or haemorrhage ? A clinical diagnosis by means of the Allen ('Guy's Hospital') score. F Neurol 1992; 239:411-3.

8 Bamford JM, Sandercock PAG, Warlow CP, Slattery J. Interobserver agreement for the assessment of handicap in stroke patients. Stroke 1989;6:828.

9 Wallace JD, Levy LL. Blood pressure after stroke. $\mathscr{f} A M A$ 1981;246:2177-80.

10 Goldstein LB and the Acute Stroke Study Investigators. Common drugs may influence motor recovery after stroke. Neurology 1995;45:865-70. 
11 O'Brien E, Mee F, Atkins N, O'Malley K. Accuracy of SpaceLabs 90207 determined by the British Hypertension Society protocol. F Hypertens 1991;9:573-4.

12 Fotherby M, Iqbal P, Potter J. Orthostatic blood pressure on prolonged standing in elderly hospital in-patients. Blood Press 1997;6:343-8.

13 Johnson RH, Smith AC, Spalding JMK, Wollner L. Effect of posture on blood pressure in elderly patients. Lancet $1965 ; 1$ : 731-3.

14 Smith JJ, Porth CM, Erickson M. Haemodynamic response to the upright posture. F Clin Pharmacol 1994;34:375-86.

15 Wieling W. Standing, orthostatic stress, and autonomic function. In: Bannister R, Mathias CJ, eds, A textbook of clinical disorders of the autonomic nervous system, 2nd edn. Oxford: Oxford University Press, 1988; p 308-20.

16 Jansen PAF, Thien T, Gribnau FW, Scurte BPM, Willemsen IJ, Lamers KJB. Blood pressure and both venous and urinary catecholamines after cerebral infarction. Clin Neurol Neurosurg 1988;90:41-5.

17 Sander D, Klingelhofe J. Changes of circadian blood pressure patterns after haemodynamic and thromboembolic ure patterns after haemodynamic and

18 Wieling W. Laboratory assessment of disturbances in cardiovascular control. In: Kenny RA, ed, Syncope in the older patient. London: Chapman and Hall, 1996; $\mathrm{p} 47-71$.

$19 \mathrm{Ng}$ AV, Callister R, Johnson DG, et al. Age and gender influence muscle sympathetic nerve activity at rest in healthy humans. Hypertension 1993;21:498-503.

20 Veith RC, Featherstone RA, Linares OA, Halter JB. Age differences in plasma norepinephrine kinetics in humans. $\mathcal{F}$ Gerontol 1986;41:319-24.

21 Robinson TG, Potter JF. Postprandial and orthostatic cardiovascular changes after acute stroke. Stroke 1995;26: 1811-6.

22 Farnworth TA, Heseltine D. The effect of postprandial hypotension on rehabilitation of the frail elderly with cerebrovascular disease. F Int Med Res 1994;22:77-84.

23 Naver HK, Blomstrand C, Wallin BG. Reduced heart rate variability after right-sided stroke. Stroke 1996;27:247-51.

24 variability after right-sided stroke. Stroke 1996;27:247-51. patients. NZ Med f 1983;96:43-5.

25 Moriguchi A, Otsuka A, Kohara K, Mikami H, Ogihara T. Evaluation of orthostatic hypotension using power spectral analysis. Am 7 Hypertens 1993;6:198-203.

26 Fotherby MD, Iqbal P. Antihypertensive therapy and orthostatic responses in elderly hospital inpatients. F Hum Hypertens 1997;11:291-4.
27 Fotherby MD, Potter JF. Orthostatic hypotension and antihypertensive treatment in the elderly. Postgrad Med 71994 ; 70:878-81.

28 Celani MG, Righett E, Migliacci R, Zampolini M, Antoniutti L, Grandi FC. Comparability and validity of two clinical scores in the early differential diagnosis of acute stroke. BMF 1994;308:1674-6.

29 Fotherby MD, Robinson TB. Reduced diurnal blood pressure variation in haemorrhagic stroke. Age Ageing 1997; 26(suppl 3):21.

30 Sander D, Klingelhofer J. Changes of circadian blood pressure patterns and cardiovascular parameters indicate lateralisation of sympathetic activation following hemispheric brain infarction. ₹ Neurol 1995;242:313-8.

31 Wieling W. Non-invasive continuous recording of heart rate and blood pressure in the evaluation of neurocardiovascular control. In: Bannister R, Mathias CJ, eds, $A$ textbook of clinical disorders of the autonomic nervous system, 3rd edn. Oxford: Oxford University Press, 1992; p 291-311.

32 Ward C, Kenny RA. Reproducibility of orthostatic hypotension in symptomatic elderly. Am F Med 1996;100:418-22.

33 Ooi WL, Barretts S, Hossain M, Kelly-Gagnon M, Lipsitz LA. Patterns of orthostatic blood pressure and their clinical correlates in a frail, elderly population. $7 A M A$ 1997;277: 1299-304.

34 Warlow CP,Dennis MS,Van Giin J, et al.Blood pressure reduction. In: Warlow CP, Dennis MS, Van Gijn J, et al, eds. Stroke: a practical guide to management. London: Blackwell Science Ltd, 1996; pp 420-1.

35 Panayiotou B, Lloyd M, Fotherby M, Crome P. Blood pressure measurement and antihypertensive therapy in acute stroke. Int f Clin Pract 1998;52:162-4.

36 Sullivan MJ, Binkley PF, Unverferth DV, Ren JH, Boudoulas H, Bashore TM. Prevention of bedrest-induced physical deconditioning by daily dobutamine infusions. F Clin Invest 1985;76:1632-42.

37 Krajewski A, Freeman R, Rutheser R. Transcranial Doppler assessment of the cerebral circulation during postprandial hypotension in the elderly. 7 Am Geriatr Soc 1993;41:19-24.

38 Mori S, Sadoshima S, Fujii K, Ibayashi S, Iino K, Fijyshima A. Decrease in cerebral blood flow with blood pressure A. Decrease in cerebral blood flow with blood pressure reduction

39 Shannon JR, Jordan J, Robertson D. Blood pressure in autonomic failure: drinks, meals and other ordeals. Clin Sci 1998;94:5 